\title{
Taxas de geração de viagens em condomínios residenciais Niterói - estudo de caso
}

\author{
Elisabeth Poubel Grieco ${ }^{1}$; Licinio da Silva Portugal ${ }^{2}$
}

\begin{abstract}
Resumo: Este artigo apresenta um estudo sobre a geração de viagens em residências, realizado em duas regiões distintas na Cidade de Niterói, Rio de Janeiro. A hipótese levantada investigou que fatores de localização e de uso do solo influenciam diretamente no padrão de viagens. O procedimento proposto foi aplicado em onze condomínios e, a partir dos dados levantados, elaboraram-se as taxas e os modelos de geração de viagens, pela regressão linear, relacionando os volumes de tráfego com as variáveis que expressam a quantidade de unidades residenciais e o número de vagas disponíveis nos empreendimentos. Complementarmente, pode-se calcular o percentual de fluxo nos horários de pico e as viagens realizadas a pé, analisando os resultados obtidos nas duas regiões e comparando-os às estimativas das viagens derivadas dos modelos americanos. Concluiu-se, por meio dos modelos de regressão, a confirmação da hipótese. Com este trabalho, espera-se poder contribuir para o estudo mais abrangente da geração de viagens em uso residencial, oferecendo aos planejadores urbanos ferramenta importante na concepção de cidades mais sustentáveis.
\end{abstract}

Abstract: This article presents a trip generation study for a residential area, at two distinct regions of the city of Niterói, state of Rio de Janeiro. The procedure was applied in eleven condominiums, and, with these data, the rates and trip generation models have been developed, using linear regression. For each region, the variables were the occupied units and the total parking spaces. Complementing the results, the percentage of flow in peak periods and the trips produced by foot were calculated. It is expected that this work may contribute for the study of trip generation in residential areas, essential tool for urban planners who wish to design more sustainable cities.

\section{INTRODUÇÃO}

Dimensionar o sistema viário e a oferta de transporte público tornou-se um grande desafio para técnicos e planejadores, principalmente nos últimos cinqüenta anos, quando o processo de urbanização se acelerou, transformando rapidamente as cidades ao redor do mundo. No Brasil, o Estatuto da Cidade (Lei $10.257 / 2001$ ) abriu importante precedente para o estudo mais aprofundado do ambiente urbano, com a previsão em lei dos Estudos de Impacto de Vizinhança, que inclui, além da preocupação com a população vizinha afetada diretamente, o tráfego que vai ser gerado e a demanda por transporte público.

Alguns municípios brasileiros, como Juiz de Fora, São Paulo e Belo Horizonte, estabeleceram, pela legislação específica, que empreendimentos comerciais, industriais ou residenciais, a partir de determinado porte, seriam considerados Pólos Geradores de Viagens (PGVs) e, neste caso, Estudos de Impacto de Vizinhança (EIVs) são exigidos para a sua aprovação. Esses estudos devem demonstrar, entre outros aspectos, que o tráfego adicional gerado pode ser absorvido pelo sistema viário do entorno e que os impactos negativos desse aumento de tráfego podem ser mitigados

\footnotetext{
${ }^{1}$ Elisabeth Poubel Grieco, Programa de Engenharia de Transportes da COPPE, Universidade Federal do Rio de Janeiro, Rio de Janeiro, RJ, Brasil. (e-mail: bethgrieco@hotmail.com).

${ }^{2}$ Licinio da Silva Portugal, Programa de Engenharia de Transportes da COPPE, Universidade Federal do Rio de Janeiro, Rio de Janeiro, RJ, Brasil. (e-mail: licinio@pet.coppe.ufrj.br).
}

Manuscrito recebido em 18/1/2010 e aprovado para publicação em 8/3/2010. Este artigo é parte de TRANSPORTES, volume XVIII, número 1, março de 2010. ISSN: 2237-1346 (online). ou compensados. Para tanto, é fundamental que se calculem as viagens que serão geradas ou atraídas pelo empreendimento em análise.

O volume de tráfego é estimado a partir de dados sobre a geração de viagens. Inúmeros estudos mostram que fatores, tais como a renda familiar, a densidade residencial, o uso e a ocupação do solo, a oferta de estacionamento, o desenho urbano, dentre outros, influenciam diretamente nos padrões de viagens, o que mostra a necessidade de taxas e modelos compatíveis com as especificidades locais.

Nesse sentido, o presente artigo propõe a determinação de modelos e taxas de geração de viagens por meio de pesquisa em condomínios residenciais, tomando como referência a similaridade da renda familiar e adotando duas tipologias construtivas (uma horizontal e outra vertical) em duas regiões da cidade de Niterói com distintos tipos de uso e ocupação solo, assumindo que eles poderão interferir no padrão de viagens.

\section{REFERENCIAL TEÓRICO}

Há vários fatores que influenciam nas viagens geradas a partir de um empreendimento. Segundo Bruton (1979), a viagem é função de três fatores básicos na área de estudo: padrão de uso do solo e do desenvolvimento da área; características socioeconômicas da população que se desloca; natureza, extensão e capacidade do sistema de transportes.

Taxas de geração de viagens são definidas como um índice calculado estatisticamente a partir de contagens feitas nos locais de interesse, portanto, são influencia- 
das diretamente pelas características de localização do empreendimento, porte e tipo de atividade ali desenvolvida.

Os Pólos Geradores de Viagens, ou simplesmente PGVs, são, segundo Portugal e Goldner (2003), “empreendimentos de distintas naturezas que têm em comum o desenvolvimento de atividades em um porte e escala capazes de gerar um contingente significativo de viagens".

Os PGVs são caracterizados segundo o seu tipo e o porte. O tipo é a finalidade do PGV, ou seja, a atividade que ali se desenvolve. O ITE (2008) denomina cada tipo de PGV como um "Uso do solo" diferenciado. O porte representa o tamanho e está diretamente ligado à magnitude da demanda de viagens que o empreendimento atrairá ou produzirá e é a variável mais utilizada nos modelos de estimativa de geração de viagens. Como exemplo, o porte pode ser calculado pela área bruta locável (ABL) dos centros comerciais ou pela quantidade de unidades ocupadas nos condomínios residenciais. Também podem ser consideradas grandezas como número de leitos de um hospital e número de alunos de uma escola.

O Institute of Transportation Engineers (ITE) dos Estados Unidos é a principal referência em modelos de geração de viagem, através de publicações, das quais ressalta-se o Trip Generation (2008), já na sua oitava edição, posterior a de 2003 ( $7^{\mathrm{a}} \mathrm{Ed}$.). É resultado de uma grande pesquisa que complilou dados levantados pela ação voluntária de pesquisadores e profissio- nais em vários estados norte-americanos. As variáveis utilizadas pelo ITE para as residências são: o número de unidades ocupadas; o número de moradores; e o número de veículos, sendo calculadas as taxas e equações de regressão para horas de pico, dias de semana, sábados e domingos.

Segundo a Rede Ibero-Americana em Estudos de Pólos Geradores de Viagens, o ITE (2004) adota três métodos para a obtenção das taxas de viagens:

1. Coleta dos dados dos términos das viagens (atraídas + produzidas) multiplicada pelo tamanho da variável independente do tipo do empreendimento pesquisado;

2. Cálculo da média ponderada do número das viagens (atraídas + produzidas) por unidade da variável independente, pelo número de unidades da variável independente associado com o empreendimento proposto;

3. Determinação de equação de regressão através de software estatístico, relacionando os dados obtidos das viagens geradas (ordenada) com os da variável independente (abscissa). Neste caso, o coeficiente de determinação $\mathrm{R}^{2}$ indicará a melhor aproximação da curva às variáveis.

O ITE (2004) sugere procedimento para a coleta de dados local na metodologia representada na Figura 1.

A geração de viagens tem padrões diferentes para cada tipo de uso do solo e também varia de acordo com o horário e o dia da semana. Neste estudo, têm-se como objeto de interesse as residências tipos Land

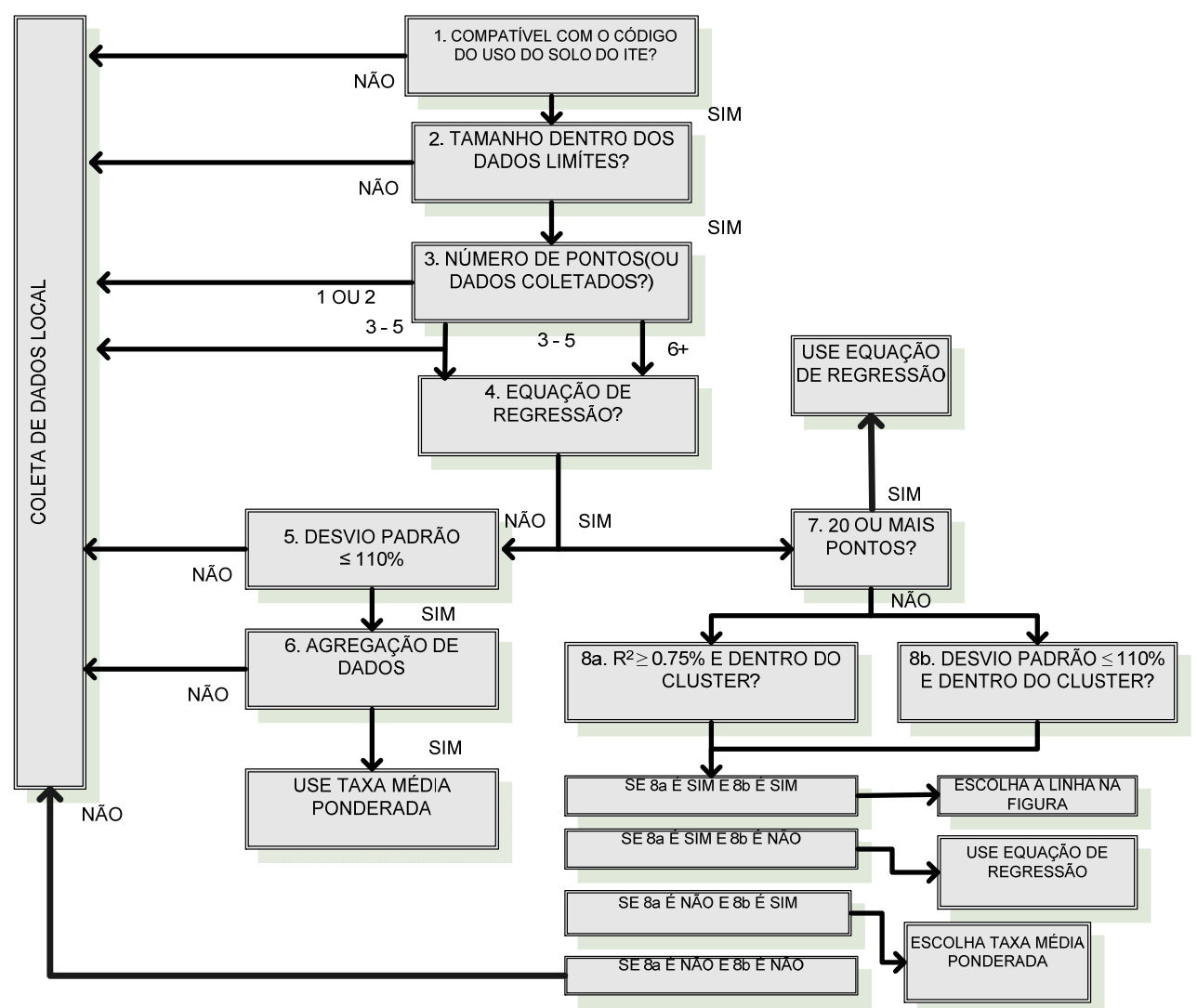

Figura 1. Procedimento recomendado pelo do ITE (2004) para a obtenção de taxas de geração e viagens 
Use (210 e 222 do Trip Generation, 2008, pp. 289 e 369 ), por serem similares aos condomínios aqui pesquisados, verificando-se que o número de veículos e a quantidade de moradores têm uma forte correlação com a média das viagens para um dia de semana e nos horários de pico.

No Brasil, o estudo da geração de viagens em residências é ainda pouco explorado, mas certamente necessário para se dispor de taxas e modelos mais compatíveis com a realidade. Goldner et al. (2006) realizaram pesquisas para hotéis na cidade de Florianópolis que indicaram que as estimativas do ITE tendem a ser superestimadas, o que também foi verificado por Andrade e Portugal (2010), no caso dos shopping centers.

A Companhia de Engenharia de Tráfego de São Paulo (CET-SP) publicou, em 1983, o Boletim Técnico número 32 , trabalho resultado de pesquisa realizada pela sua equipe técnica. Os modelos de geração de viagens foram direcionados principalmente a lojas, hospitais, escolas, indústrias e supermercados. Embora não tenham dados locais sobre geração de viagens, algumas cidades têm se preocupado quanto à definição de parâmetros que caracterizam um empreendimento como PGV, como também com a regulamentação do procedimento de análise. A cidade de Niterói definiu que empreendimentos residenciais com área superior a $25.000 \mathrm{~m}^{2}$ dependem da elaboração de Estudos de Impacto de Vizinhança para sua aprovação, regulamentado através da Lei 2050/2003. Cunha (2009) destaca a legislação pertinente e a sistemática de aprovação dos PGVs nas cidades de Belo Horizonte, São Paulo, Curitiba, Juiz de Fora e Rio de Janeiro, sintetizando-se na Tabela 1 os parâmetros adotados nos empreendimentos residenciais, segundo o ITE 2005 e algumas cidades pesquisadas pelo autor.
Tabela 1. Parâmetros de definição de PGV em algumas cidades, adaptado de Cunha (2009)

\begin{tabular}{lll}
\hline & \multicolumn{1}{l}{ Casa } & 90 unidades \\
\cline { 2 - 3 } ITE (2005) & Apartamentos & 150 unidades \\
\cline { 2 - 3 } & Condomínios & 190 unidades \\
\hline \multirow{3}{*}{ Belo Horizonte } & Multifamiliar & $\geq 150$ unidades \\
\cline { 2 - 3 } & Uso misto & $\begin{array}{l}\geq 150 \text { unidades } \\
\text { residencias }+ \\
\text { comercial }=6000 \mathrm{~m}^{2}\end{array}$ \\
\hline \multirow{3}{*}{ Juiz de Fora } & $\begin{array}{l}\text { Residencial } \\
\text { vertical }\end{array}$ & $\geq 100$ unidades \\
\cline { 2 - 3 } & $\begin{array}{l}\text { Residencial } \\
\text { horizontal }\end{array}$ & $\geq 50$ unidades \\
\hline Rio de Janeiro & Residencial & $\geq 200$ unidades \\
\hline
\end{tabular}

O presente estudo, além de estabelecer as taxas de geração de viagens, pretende avaliar a influência que o uso e a ocupação do solo têm sobre a geração de viagens pela comparação de dados obtidos em pesquisa realizada simultaneamente em duas regiões de Niterói com diferentes padrões de urbanização. Foram comparados dados coletados na região de Pendotiba, que tem baixa densidade, uso exclusivamente residencial e tipologia horizontal; e em Icaraí, que, contrariamente, tem alta densidade, uso diversificado e tipologia vertical.

\section{PROCEDIMENTO PROPOSTO}

A metodologia utilizada foi derivada da adotada pelo ITE (2004) e aplicada na cidade de Niterói, localizada na Região Metropolitana do Rio de Janeiro, com uma população de 459.451 habitantes, segundo o Censo

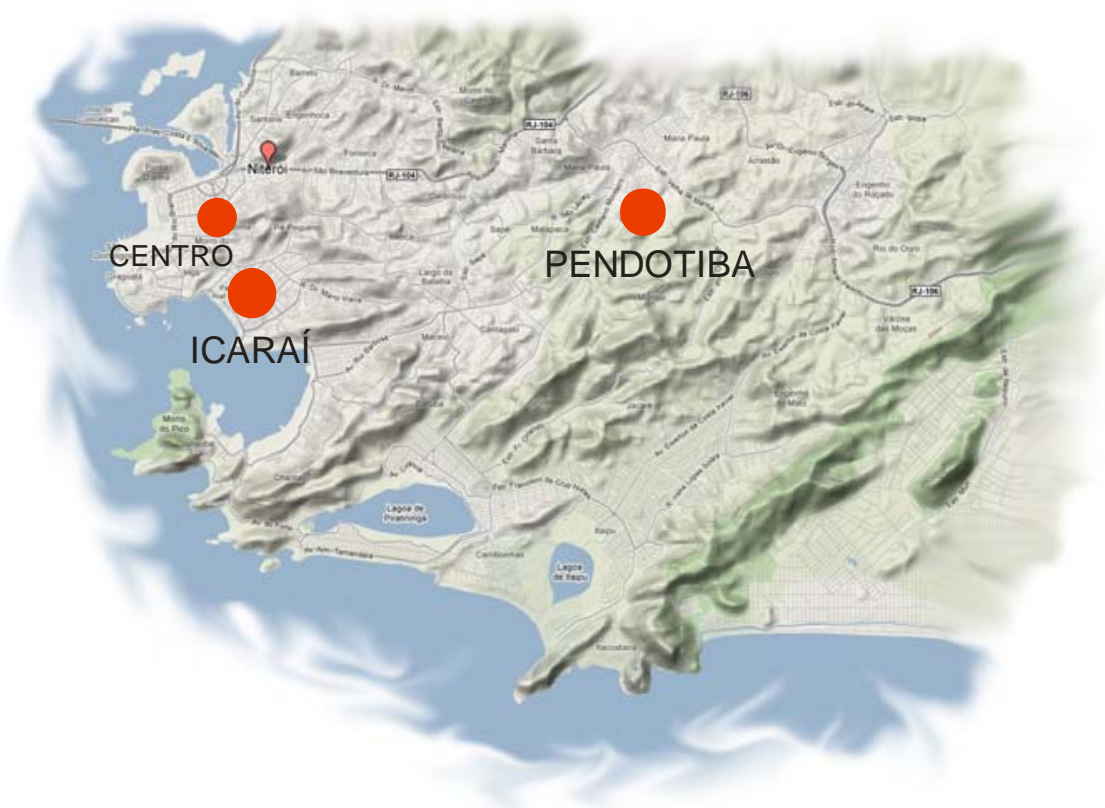

Fiqura 2. Mapa de Niterói com a localizacão das áreas de estudo e o Centro 
Demográfico de 2000, estimada em 477.151 habitantes em 2007, e uma área de $134,5 \mathrm{~km}^{2}$ (ver Figura 2), o que faz de Niterói o quinto município mais populoso do Estado do Rio de Janeiro. Segundo o Detran-RJ, a frota de veículos particulares em 2007, na cidade de Niterói, era de 161.630 automóveis, calculando-se o índice de motorização neste ano de 33,87 veículos para cada100 habitantes. Pode-se comparar com o índice de motorização médio do Brasil que, neste mesmo ano, era de 15,80 veículos/100 habitantes (praticamente metade do da cidade de Niterói), e dos Estados Unidos (EUA), de 45,10 veículos/100 habitantes (IRF World Road Statistics, 2009), isto é, 33\% superior ao de Niterói.

Como o trabalho se propõe a definir as taxas de geração de viagens de duas tipologias diferentes de ocupação na cidade de Niterói, a escolha dos locais para o levantamento dos dados foi baseado nos seguintes atributos (ver Figura 3):

- Densidade - expressa por habitante por quilômetro quadrado ( $\mathrm{hab} / \mathrm{km}^{2}$ ). As duas regiões têm características propositalmente opostas em relação à densidade. Enquanto se observa alta concentração populacional em Icaraí, Pendotiba se caracteriza pelo espalhamento da ocupação em baixa densidade;

- Renda - foram escolhidos condomínios de renda equivalente para que esse atributo não viesse a distorcer o resultado da pesquisa, já que se busca definir taxas e comparar o padrão de mobilidade ligado apenas aos fatores relativos ao uso e ocupação do solo;

- Uso do Solo - as duas regiões apresentam grande diversidade quanto à tipologia construtiva, gabarito, tamanho dos lotes e sistema viário. São também diferentes intencionalmente quanto a sua tipologia: a região 1 (Icaraí) tem rica mistura de usos, comércio local diversificado e acessível e facilidade para transporte público; a região 2 (Pendotiba) apresenta predominância residencial horizontal, com pouca acessibilida-

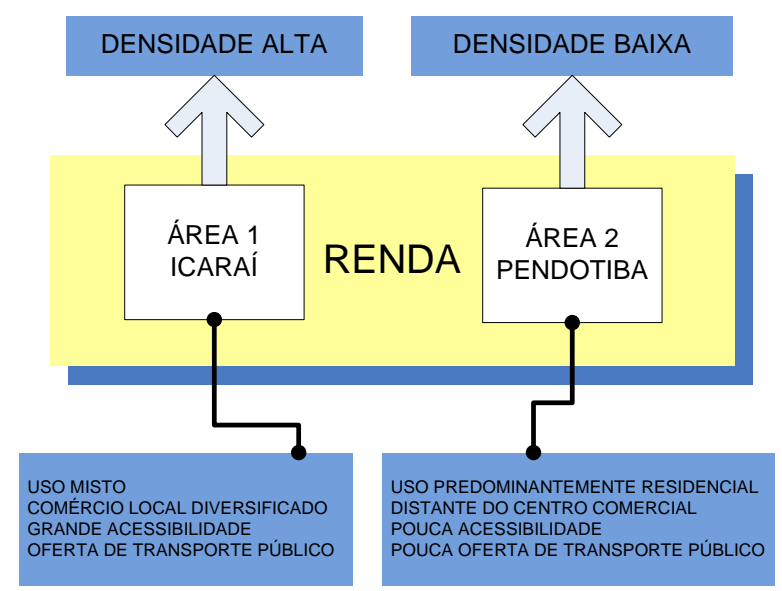

Figura 3. Atributos considerados na metodologia de aos serviços e ao transporte coletivo.

O IBGE, no censo de 2000, coletou dados dos setores censitários e, com base nessas informações, foi possível identificar os setores que mais se enquadravam para a aplicação da pesquisa em campo. As características das regiões são peculiares e nelas predominam:

- Em Icaraí: condomínios verticais, com alta densidade, em média $126.000 \mathrm{hab} / \mathrm{km}^{2}$; uso misto e significativa oferta de transporte público. Operam no bairro oito empresas de transporte coletivo, com 14 linhas municipais e seis linhas intermunicipais, que ligam Icaraí ao Centro de Niterói e ao Rio de Janeiro. Dos 1905 táxis autorizados na cidade, 207 carros lá circulam, utilizando 36 pontos de apoio (PA) espalhados pelo Bairro;

- Em Pendotiba: condomínios horizontais de baixa densidade, em média $2.074 \mathrm{hab} / \mathrm{km}^{2}$, com uso estritamente residencial. O transporte público nessa região é operado por uma única empresa, que dispõe de duas linhas municipais que têm por destino o Centro da cidade e uma linha intermunicipal que liga a região ao Centro do Rio de Janeiro. Não dispõe de um centro de comércio mais elaborado. O serviço de táxi é feito por central de telefone com tarifa diferenciada.

Foi considerado que em cada região deve ter um mínimo de 5 observações, ou seja, no mínimo 5 condomínios devem ser analisados, buscando parâmetros que atendam ao recomendado pelo ITE (2004), isto é : $\mathrm{R}^{2} \geq 0,75$ e Desvio Padrão $\leq 110 \%$ (ver Figura 1 ). Para a região de Pendotiba, foram pesquisados cinco condomínios e na Região de Icaraí, seis condomínios.

A Tabela 2 numera os condomínios e relaciona as variáveis que foram utilizadas neste estudo. Na região de Pendotiba, o maior condomínio pesquisado tem 171 unidades residenciais e o menor, 15 , com média de 69 unidades por condomínio. Em Icaraí, o maior tem 254 unidades e o menor, 48, com média de 136 unidades por condomínio. Dos 11 condomínios pesquisados, segundo Cunha (2009), apenas quatro estão abaixo do valor mínimo para ser considerado um PGV, conforme parâmetros desenvolvidos para outras cidades, em função da expectativa da geração de viagens (Tabela 1).

Na média, o porte dos condomínios é bem inferior (cerca de um terço, em termos de número de unidades residenciais ocupadas) à média dos pesquisados pelo ITE (2008), mas os valores estão predominantemente dentro do intervalo considerado, o que indica a observância do cluster (ver Tabela 2 e Figura 4). De qualquer maneira, os resultados obtidos nas duas regiões apresentaram $R^{2} \geq 0,75$, o que atende, portanto, a esse 
Tabela 2. Lista dos condomínios por tamanho das variáveis

\begin{tabular}{|c|c|c|c|c|}
\hline & No. & Condomínio & $\begin{array}{l}\text { Número de unidades } \\
\text { residenciais (UR) }\end{array}$ & $\begin{array}{c}\text { Número de vagas } \\
(N V)\end{array}$ \\
\hline \multirow{6}{*}{ 胥 } & 1 & Villa España & 112 & 132 \\
\hline & 2 & Vila Real & 168 & 95 \\
\hline & 3 & Fra Angelico & 68 & 72 \\
\hline & 4 & Costa do Sol & 254 & 340 \\
\hline & 5 & Camargue e Provence & 168 & 171 \\
\hline & 6 & Ed. Rogério & 48 & 48 \\
\hline \multirow{5}{*}{ 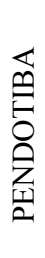 } & 7 & Belo Vale & 48 & $95^{*}$ \\
\hline & 8 & Vila Verde & 44 & $90 *$ \\
\hline & 9 & Jardim Pendotiba & 15 & $30^{*}$ \\
\hline & 10 & Ubá Curumim & 67 & $135^{*}$ \\
\hline & 11 & Ubá Pendotiba & 171 & $540^{*}$ \\
\hline
\end{tabular}

\section{critério.}

Para a determinação das taxas, foram adotados como variáveis explicativas o número de unidades residenciais (UR) ocupadas e o número de vagas oferecidas (NV). A escolha dessas variáveis partiu da facilidade de serem obtidas nesse procedimento, como também por determinar o porte dos estabelecimentos. A Tabela 3 compara as taxas observadas na pesquisa com as taxas obtidas no manual do ITE (2008), relacionando a média de unidades residenciais de cada região, suas variáveis e a densidade dos setores em que os condomínios estão localizados.

O horário para o início das contagens foi $06 \mathrm{~h} 30 \mathrm{~min}$, levando em conta que as viagens geradas pelo tipo do uso residencial são viagens origens (se iniciam a partir das residências pela manhã) até as $20 \mathrm{~h} 00 \mathrm{~min}$, o que permitiu observar as atividades rotineiras dos moradores dos condomínios investigados. A pesquisa foi realizada em dias de uma semana típica (4 ${ }^{\mathrm{a}}$. e $5^{\mathrm{a}}$. feira, sem feriado).

Como forma de se obter informações sobre o número de viagens por automóveis geradas nos condomínios escolhidos, foi elaborada uma planilha para a contagem dos movimentos de veículos. Observa-se que, além das viagens geradas por automóveis, foram contadas as viagens de pessoas que entraram e saíram a pé dos condomínios nos mesmos intervalos de tempo, com o objetivo de se calcular também as taxas de viagens geradas, pressupondo que elas abrangem não só as viagens a pé, mas também as viagens por transporte público, o que será aqui denominado como viagens de pessoas, distinguindo-as das associadas aos automóveis.

\section{APRESENTAÇÃO DOS RESULTADOS}

Após as contagens veiculares e de pessoas a pé, os dados registrados por condomínio são totalizados de maneira a gerar as quantidades de viagens diárias, apresentadas nas Tabelas 4, 5 e 6 . Para a determinação das taxas médias, foi considerado o seguinte procedimento:

a) Totalização do número de viagens por automóveis e viagens de pessoas (entradas e saídas) para cada condomínio;

b) Cálculo da taxa de entrada e saída para cada condomínio e a taxa total de cada condomínio para cada variável explicativa;

c) Cálculo da taxa média ponderada com as variáveis explicativas: unidades residenciais (UR) e número de vagas (NV) para cada movimento (entrada e saída) na região estudada;

d) Cálculo do desvio padrão entre a taxa média obtida e a taxa individualizada.

\subsection{Viagens por automóveis}

Com as planilhas de contagens, pode-se calcular o percentual dos movimentos de entrada e saída dos au-

Tabela 3. Variáveis explicativas e a densidade dos setores censitários dos condomínios

\begin{tabular}{lcccccccc}
\hline \multicolumn{4}{c}{ Estudo do ITE } & \multicolumn{3}{c}{ Estudo proposto } \\
\hline & Uso do solo & $\begin{array}{c}\text { No. médio } \\
\text { de unidades } \\
\text { ocupadas }\end{array}$ & $\begin{array}{l}\text { No. médio } \\
\text { de vagas* }\end{array}$ & $\begin{array}{c}\text { Taxa média } \\
\text { diária }\end{array}$ & $\begin{array}{c}\text { No. médio } \\
\text { de unidades } \\
\text { ocupadas }\end{array}$ & $\begin{array}{c}\text { No. médio } \\
\text { de vagas }\end{array}$ & $\begin{array}{c}\text { Taxa média } \\
\text { diária }\end{array}$ & $\begin{array}{c}\text { Densidade } \\
\text { média do } \\
\text { setor }\end{array}$ \\
\hline ICARAÍ & $222, p g 369$ & 456 & $\mathrm{X}$ & 4,20 & 136 & 143 & 1,39 & 126,822 \\
\hline PENDOTIBA & $210, p g 290$ & 197 & 257 & 9,57 & 69 & 178 & 5,10 & 2,074 \\
\hline
\end{tabular}


Tabela 4. Viagens Diárias por automóveis

REGIÃO DE ICARAÍ

\begin{tabular}{|c|c|c|c|c|c|c|c|c|c|c|c|}
\hline \multirow{2}{*}{ CONDOMÍNIO } & \multicolumn{3}{|c|}{ NO. DE VIAGENS } & \multirow{2}{*}{$\begin{array}{c}\text { UR- NO. DE } \\
\text { UNIDADES } \\
\text { RESIDENCIAIS }\end{array}$} & \multirow{2}{*}{$\begin{array}{c}\text { TAXA } \\
\text { ENTRADAS } \\
\text { UR }\end{array}$} & \multirow{2}{*}{$\begin{array}{c}\text { TAXA } \\
\text { SAÍDAS/UR }\end{array}$} & \multirow{2}{*}{$\begin{array}{c}\text { TAXA } \\
\text { UR/TOTAL }\end{array}$} & \multirow{2}{*}{$\begin{array}{c}\text { NV - } \\
\text { NÚMERO } \\
\text { DE VAGAS }\end{array}$} & \multirow{2}{*}{$\begin{array}{c}\text { TAXA } \\
\text { ENT RADAS/NV }\end{array}$} & \multirow{2}{*}{$\begin{array}{c}\text { TAXA } \\
\text { SAÍDAS/NV }\end{array}$} & \multirow{2}{*}{$\begin{array}{c}\text { TAXA } \\
\text { NV/TOTAL }\end{array}$} \\
\hline & ENTRADAS & SAÍDAS & TOTAL & & & & & & & & \\
\hline 1 & 122 & 176 & 298 & 110 & 1,11 & 1,60 & 2,71 & 160 & 0,76 & 1,10 & 1,86 \\
\hline 2 & 61 & 62 & 123 & 168 & 0,36 & 0,37 & 0,73 & 95 & 0,64 & 0,65 & 1,29 \\
\hline 3 & 47 & 42 & 89 & 68 & 0,69 & 0,62 & 1,31 & 72 & 0,65 & 0,58 & 1,24 \\
\hline 4 & 177 & 228 & 405 & 254 & 0,70 & 0,90 & 1,59 & 340 & 0,52 & 0,67 & 1,19 \\
\hline 5 & 69 & 94 & 163 & 168 & 0,41 & 0,56 & 0,97 & 171 & 0,40 & 0,55 & 0,95 \\
\hline 6 & 23 & 35 & 58 & 48 & 0,48 & 0,73 & 1,21 & 48 & 0,48 & 0,73 & 1,21 \\
\hline \multicolumn{2}{|c|}{ DESVIO PADRÃO\% } & & & 76,18 & 0,28 & 0,43 & 0,70 & 105,94 & 0,13 & 0,20 & 0,30 \\
\hline \multicolumn{3}{|c|}{ MÉDIA PONDERADA } & & 136 & 0,61 & 0,78 & 1,39 & 148 & 0,56 & 0,72 & 1,28 \\
\hline \multicolumn{12}{|c|}{ REGIÃO DE PENDOTIBA } \\
\hline \multirow{2}{*}{$\begin{array}{c}\text { NO. DO } \\
\text { CONDOMÍNIO }\end{array}$} & \multicolumn{3}{|c|}{ NO. DE VIAGENS } & \multirow{2}{*}{\begin{tabular}{|c|} 
UR- NO. DE \\
UNIDADES \\
RESIDENCIAIS \\
\end{tabular}} & \multirow{2}{*}{$\begin{array}{c}\text { TAXA } \\
\text { ENT RADAS } \\
\text { UR } \\
\end{array}$} & \multirow{2}{*}{$\begin{array}{c}\text { TAXA } \\
\text { SAÍDAS/UR }\end{array}$} & \multirow{2}{*}{$\begin{array}{c}\text { TAXA } \\
\text { UR/TOTAL }\end{array}$} & \multirow{2}{*}{\begin{tabular}{|c|} 
NV - \\
NÚMERO \\
DE VAGAS \\
\end{tabular}} & \multirow{2}{*}{$\begin{array}{c}\text { TAXA } \\
\text { ENT RADAS/NV }\end{array}$} & \multirow{2}{*}{$\begin{array}{c}\text { TAXA } \\
\text { SAÍDASINV }\end{array}$} & \multirow{2}{*}{$\begin{array}{c}\text { TAXA } \\
\text { NV/TOTAL }\end{array}$} \\
\hline & ENT RADAS & SAÍDAS & TOTAL & & & & & & & & \\
\hline 7 & 122 & 126 & 248 & 48 & 2,54 & 2,63 & 5,17 & 96 & 1,27 & 1,31 & 2,58 \\
\hline 8 & 129 & 138 & 267 & 44 & 2,93 & 3,14 & 6,07 & 88 & 1,47 & 1,57 & 3,03 \\
\hline 9 & 54 & 59 & 113 & 15 & 3,60 & 3,93 & 7,53 & 30 & 1,80 & 1,97 & 3,77 \\
\hline 10 & 189 & 211 & 400 & 67 & 2,82 & 3,15 & 5,97 & 134 & 1,41 & 1,57 & 2,99 \\
\hline 11 & 371 & 360 & 731 & 171 & 2,17 & 2,11 & 4,27 & 342 & 1,08 & 1,05 & 2,14 \\
\hline \multicolumn{3}{|c|}{ DESVIO PADRÃO\% } & & 59,98 & 0,53 & 0,68 & 1,21 & 119,96 & 0,26 & 0,34 & 0,60 \\
\hline \multicolumn{3}{|c|}{ MÉDIA PONDERADA } & & 69,00 & 2,51 & 2,59 & 5,10 & 138,00 & 1,25 & 1,30 & 2,55 \\
\hline
\end{tabular}

tomóveis dos empreendimentos nos horários de pico do tráfego local (Tabela 4) nas duas regiões, possibilitando, assim, calcular as taxas de viagens geradas, como também o percentual de entrada/saída nos horários de pico nos condomínios e a taxa média por variável em cada região (Tabela 5). Percebe-se que na região de Icaraí o fluxo mais intenso de viagens ocorre no pico da tarde, ao passo que, em Pendotiba, a quantidade de viagens nos dois horários é igual.

Observa-se que, na região de Pendotiba, o desvio padrão referente à variável UR é ligeiramente superior aos $110 \%$ com o $\mathrm{R}^{2} \geq 0,75$ e, neste caso, pode-se indicar o uso da equação de regressão para obtenção da estimativa de viagens (ITE, 2004).

Os resultados obtidos nas contagens permitem a comparação entre as taxas de viagens geradas e as variáveis em cada local pesquisado. Na média, as taxas das duas regiões são diferentes.
Quando comparados os volumes com a variável número de unidades, nota-se que em Pendotiba os valores das taxas são bem acima das de Icaraí. Por exemplo, na Tabela 3, comparando os condomínios $3 \mathrm{e}$ 10, a variável UR é praticamente a mesma e as suas respectivas taxas de geração de viagens são bem diferentes. Para melhor ilustrar a comparação entre as taxas obtidas em relação ao porte do empreendimento nas duas regiões, observa-se a Figura 4, podendo-se então afirmar que:

- Existe grande diferença das taxas entre as regiões (Pendotiba - curva em vermelho e Icaraí curva em azul);

- O porte do empreendimento aqui expresso pelas variáveis UR pouco influi no resultado das taxas obtidas;

- O que mais afetou a geração de viagens por autos foi a localização do empreendimento e a sua

Tabela 5. Viagens por automóveis nos horários de pico e percentuais de entrada e saídas

\begin{tabular}{|c|c|c|c|c|c|c|c|c|c|c|c|c|c|c|}
\hline \multicolumn{15}{|c|}{ REGIÃO DE ICARAÍ } \\
\hline \multirow[b]{2}{*}{ CONDOMÍNIO } & \multicolumn{7}{|c|}{ PICO DA MANHÃ(6:30-9:00) } & \multicolumn{7}{|c|}{ PICO DA TARDE(16:00-19:00) } \\
\hline & ENTRADAS & SAÍDAS & TOTAL & $\begin{array}{c}\operatorname{taxa} / \text { UR } \\
\text { manhã }\end{array}$ & $\begin{array}{c}\operatorname{taxa} / \mathrm{NV} \\
\operatorname{manhã}\end{array}$ & \%ENTRADAS & $\%$ SAÍDA & ENT RADAS & SAÍDAS & TOTAL & $\begin{array}{c}\operatorname{taxa} / \mathrm{UR} \\
\text { tarde }\end{array}$ & taxa/NV tarde & $\%$ ENT RADA & \% SAÍDAS \\
\hline 1 & 6 & 47 & 53 & 0,48 & 0,33 & 11,32 & 88,68 & 37 & 35 & 72 & 0,65 & 0,45 & 51,39 & 48,61 \\
\hline 2 & 3 & 18 & 21 & 0,13 & 0,22 & 14,29 & 85,71 & 26 & 14 & 40 & 0,24 & 0,42 & 65,00 & 35,00 \\
\hline 3 & 4 & 12 & 16 & 0,24 & 0,22 & 25,00 & 75,00 & 13 & 15 & 28 & 0,41 & 0,39 & 46,43 & 53,57 \\
\hline 4 & 13 & 73 & 86 & 0,34 & 0,25 & 15,12 & 84,88 & 50 & 33 & 83 & 0,33 & 0,24 & 60,24 & 39,76 \\
\hline 5 & 2 & 24 & 26 & 0,15 & 0,15 & 7,69 & 92,31 & 27 & 16 & 43 & 0,26 & 0,25 & 62,79 & 37,21 \\
\hline 6 & 0 & 8 & 8 & 0,17 & 0,17 & 0,00 & 100,00 & 6 & 3 & 9 & 0,19 & 0,19 & 66,67 & 33,33 \\
\hline \multicolumn{2}{|c|}{ DESVIO PADRÃO $\%$} & & & 0,14 & 0,06 & & & & & & 0,17 & 0,11 & & \\
\hline MÉDIA POND. & 4,67 & 30,33 & 35 & 0,26 & 0,20 & 15,10 & 84,90 & 26,50 & 19,33 & 45,83 & 0,34 & 0,31 & 56,88 & 43,12 \\
\hline \multicolumn{15}{|c|}{ REGIÃO DE PENDOTIBA } \\
\hline \multirow{2}{*}{ CONDOMÍNIO } & \multicolumn{7}{|c|}{ PICO DA MANHÃ(6:30-9:00) } & \multicolumn{7}{|c|}{ PICO DA TARDE(16:00-19:00) } \\
\hline & ENTRADAS & SAÍDAS & TOTAL & $\operatorname{taxa} / \mathrm{UR}$ & $\operatorname{taxa} / \mathrm{NV}$ & $\%$ ENTRADA & $\%$ SAÍDA & ENT RADAS & SAÍDAS & TOTAL & $\operatorname{taxa} / \mathrm{UR}$ & $\operatorname{taxa} / \mathrm{NV}$ & $\%$ ENT RADA & \%SAÍDAS \\
\hline 7 & 18 & 37 & 55 & 1,15 & 0,57 & 32,73 & 67,27 & 37 & 19 & 56 & 1,17 & 0,58 & 66,07 & 33,93 \\
\hline 8 & 16 & 36 & 52 & 1,18 & 0,59 & 30,77 & 69,23 & 43 & 27 & 70 & 1,59 & 0,80 & 61,43 & 38,57 \\
\hline 9 & 11 & 17 & 28 & 1,87 & 0,93 & 39,29 & 60,71 & 14 & 8 & 22 & 1,47 & 0,73 & 63,64 & 36,36 \\
\hline 10 & 38 & 65 & 103 & 1,54 & 0,77 & 36,89 & 63,11 & 48 & 30 & 78 & 1,16 & 0,58 & 61,54 & 38,46 \\
\hline 11 & 51 & 112 & 163 & 0,95 & 0,48 & 31,29 & 68,71 & 104 & 73 & 177 & 1,04 & 0,52 & 58,76 & 41,24 \\
\hline DESVIO PADR & $\overline{\mathrm{A} O} \%$ & & & 0,36 & 0,18 & & & & & & 0,23 & 0,12 & & \\
\hline MÉDIA POND. & 26,8 & 53,4 & 80,2 & 1,16 & 0,58 & 33,70 & 66,33 & 49,2 & 31,4 & 80,6 & 1,17 & 0,58 & 61,14 & 38,60 \\
\hline
\end{tabular}


tipologia;

- Em Icaraí, a taxa é praticamente constante e, em Pendotiba, observa-se que a taxa tende a reduzir com o aumento do porte do condomínio.

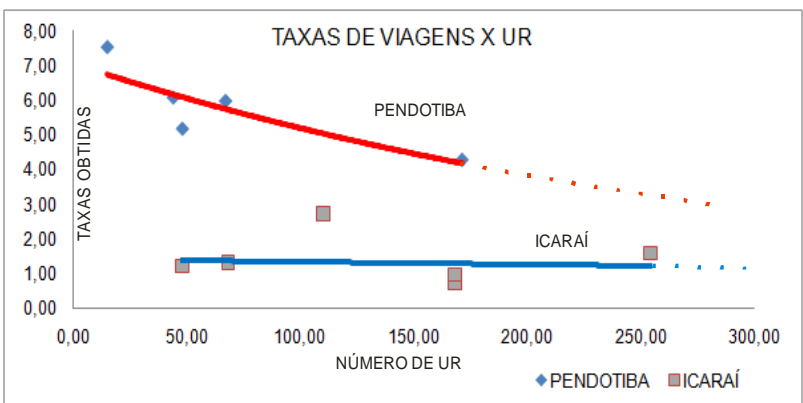

Figura 4. Taxas de geração de viagens $x$ unidades residenciais

Também se pode afirmar que existe grande diferença entre as taxas obtidas com as propostas pelo ITE, ressaltando-se que as curvas e equações se mantiveram as mesmas nas duas últimas edições do ITE de 2003 e 2008. A Figura 5 compara as curvas das taxas obtidas com as apresentadas pelo ITE (2008). Nota-se que a ocupação linear com densidades baixas, que é o caso de Pendotiba e o uso do solo 210 (ITE, 2008), apresenta curvas de tendência crescentes, enquanto curvas apresentadas pelo tipo de ocupação verticalizado com densidades altas (Icaraí e uso do solo 222) também são crescentes, porém, com menor coeficiente angular.

Ressalta-se que no ITE a amostra de 351 estabelecimentos residenciais do tipo 210 contempla condomínios com tamanho de até 3.000 unidades, mas a maioria se concentra em até 300 unidades. Já para o uso do solo 222, os 9 estabelecimentos que compõem a amostra variam em tamanho de aproximadamente 250 a 950 unidades, 8 dos quais não ultrapassam as 600 unidades.

\subsection{Viagens de pessoas}

Com as contagens das pessoas que entraram e saíram a pé de cada condomínio, calcularam-se as taxas de viagens de pessoas a partir de cada empreendimento. $\mathrm{Na}$ região de Pendotiba, só foi possível a contagem desse modal em dois condomínios. A Tabela 6 apresenta o resultado obtido e utiliza o mesmo procedimento das viagens por automóvel para determinar as taxas de viagens geradas por pessoas (a pé e por transporte público).

Ao contrário do modo automóvel, a quantidade de viagens de pessoas é mais freqüente na região de Icaraí, sendo calculada a taxa média diária de 7,04 viagens de pessoas por unidade residencial, bastante superior à da região de Pendotiba, equivalente a 1,35 por unidade residencial. Da mesma forma, podem-se comparar os condomínios 3 e 10: eles possuem 68 e 67 unidades residenciais e a taxa obtida nas viagens geradas por pessoas é de 10,06 e 1,84 respectivamente. Analisando a correlação entre as variáveis, no caso do condomínio 2 (taxa de 12,77 viagens/ vaga de estacionamento), a produção de viagens de pessoas é superior aos outros condomínios, em que a oferta de vagas é igual ou maior que a quantidade de apartamentos.

Para as taxas de viagens de pessoas (Tabela 6), conclui-se que:

- A oferta de vagas (NV) tem influência direta no padrão de viagens;

- Comparando as duas regiões, a oferta de trans-

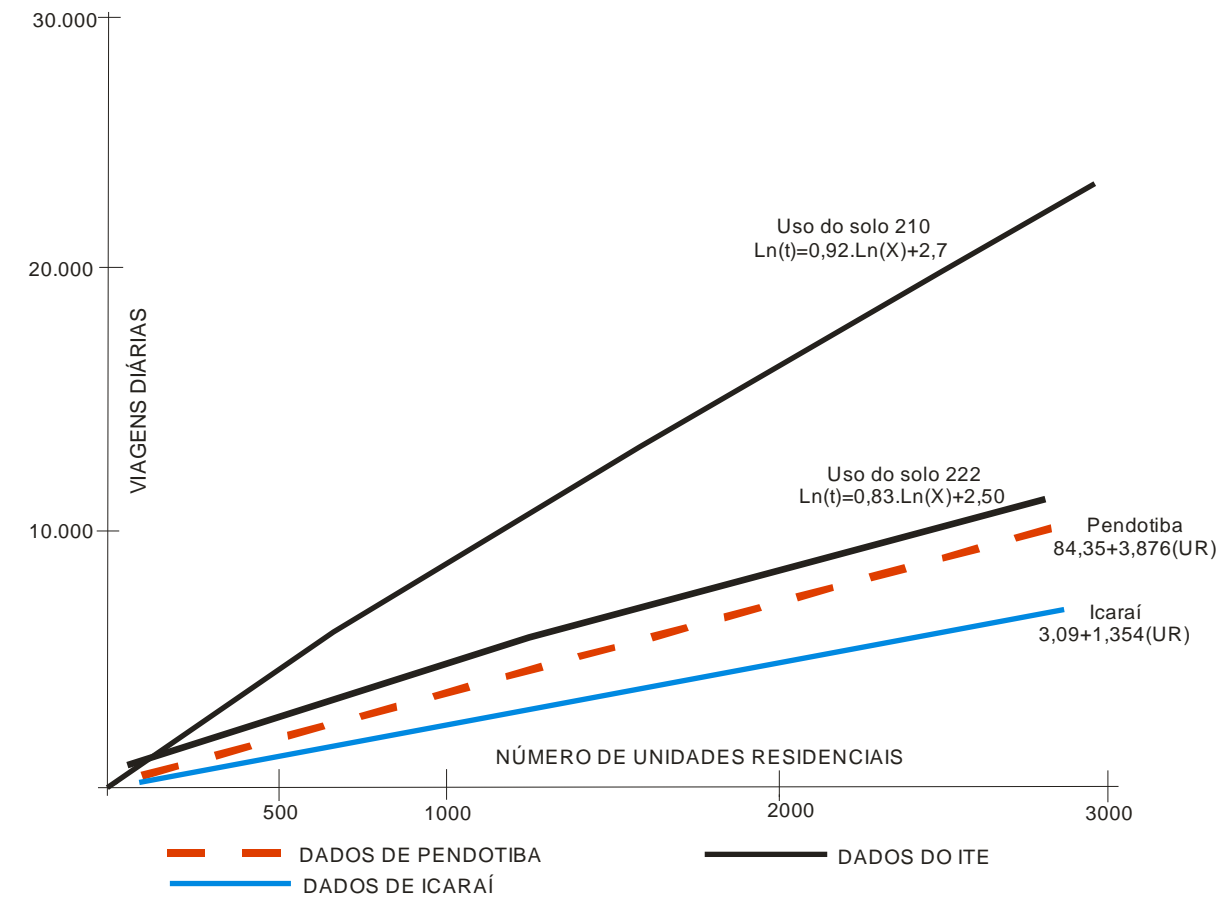

Figura 5. Relação das viagens diárias $\mathrm{X}$ número de unidades residenciais 
Tabela 6. Viagens diárias de pessoas

\begin{tabular}{|c|c|c|c|c|c|c|c|c|c|c|c|}
\hline \multicolumn{12}{|c|}{ REGIÃO DE ICARAÍ } \\
\hline \multirow{2}{*}{$\begin{array}{c}\text { NO. DO } \\
\text { CONDOMÍNIO }\end{array}$} & \multicolumn{3}{|c|}{ NO. DE VIAGENS } & \multirow{2}{*}{\begin{tabular}{|c|} 
UR- NO. DE \\
UNIDADES \\
RESIDENCIAIS
\end{tabular}} & \multirow{2}{*}{$\begin{array}{c}\text { TAXA } \\
\text { ENT RADAS/ } \\
\text { UR }\end{array}$} & \multirow{2}{*}{$\begin{array}{c}\text { TAXA } \\
\text { SAÍDAS/UR }\end{array}$} & \multirow{2}{*}{$\begin{array}{c}\text { TAXA } \\
\text { UR/TOTAL }\end{array}$} & \multirow{2}{*}{$\begin{array}{c}\mathbf{N V}- \\
\text { NÚMERO } \\
\text { DE VAGAS }\end{array}$} & \multirow{2}{*}{$\begin{array}{c}\text { TAXA } \\
\text { ENTRADASINV }\end{array}$} & \multirow{2}{*}{$\begin{array}{c}\text { TAXA } \\
\text { SAÍDAS/NV }\end{array}$} & \multirow{2}{*}{$\begin{array}{c}\text { TAXA } \\
\text { NV/TOT AL }\end{array}$} \\
\hline & ENTRADAS & SAÍDAS & TOTAL & & & & & & & & \\
\hline 1 & 511 & 432 & 943 & 110 & 4,65 & 3,93 & 8,57 & 160 & 3,19 & 2,70 & 5,89 \\
\hline 2 & 587 & 579 & 1166 & 168 & 3,49 & 3,45 & 6,94 & 95 & 6,18 & 6,09 & 12,27 \\
\hline 3 & 349 & 335 & 684 & 68 & 5,13 & 4,93 & 10,06 & 72 & 4,85 & 4,65 & 9,50 \\
\hline 4 & 769 & 907 & 1676 & 254 & 3,03 & 3,57 & 6,60 & 340 & 2,26 & 2,67 & 4,93 \\
\hline 5 & 380 & 485 & 865 & 168 & 2,26 & 2,89 & 5,15 & 171 & 2,22 & 2,84 & 5,06 \\
\hline 6 & 194 & 214 & 408 & 48 & 4,04 & 4,46 & 8,50 & 48 & 4,04 & 4,46 & 8,50 \\
\hline \multicolumn{3}{|c|}{ DESVIO PADRÃO\% } & & 76,18 & 1,06 & 0,73 & 1,75 & 105,94 & 1,55 & 1,40 & 2,92 \\
\hline \multicolumn{4}{|c|}{ MÉDIA PONDERADA } & 136 & 3,42 & 3,62 & 7,04 & 148 & 3,15 & 3,33 & 6,48 \\
\hline \multicolumn{12}{|c|}{ REGIÃO DE PENDOTIBA } \\
\hline \multirow{2}{*}{$\begin{array}{c}\text { NO. DO } \\
\text { CONDOMÍNIO }\end{array}$} & \multicolumn{3}{|c|}{ NO. DE VIAGENS } & UR- NO. DE & \multirow{2}{*}{$\begin{array}{c}\text { TAXA } \\
\text { ENT RADAS/ } \\
\text { UR }\end{array}$} & \multirow{2}{*}{$\begin{array}{c}\text { TAXA } \\
\text { SAÍDAS/UR }\end{array}$} & \multirow{2}{*}{$\begin{array}{c}\text { TAXA } \\
\text { UR/TOT AL }\end{array}$} & \multirow{2}{*}{$\begin{array}{c}\mathbf{N V}- \\
\text { NÚMERO } \\
\text { DE VAGAS }\end{array}$} & \multirow{2}{*}{$\begin{array}{c}\text { TAXA } \\
\text { ENTRADAS/NV }\end{array}$} & \multirow{2}{*}{$\begin{array}{c}\text { TAXA } \\
\text { SAÍDAS/NV }\end{array}$} & \multirow{2}{*}{$\begin{array}{c}\text { TAXA } \\
\text { NV/TOT AL }\end{array}$} \\
\hline & ENTRADAS & SAÍDAS & TOTAL & $\begin{array}{l}\text { UNIDADES } \\
\text { RESIDENCIAIS }\end{array}$ & & & & & & & \\
\hline 10 & 65 & 58 & 123 & 67 & 0,97 & 0,87 & 1,84 & 134 & 0,49 & 0,43 & 0,92 \\
\hline 11 & 112 & 87 & 199 & 171 & 0,65 & 0,51 & 1,16 & 342 & 0,33 & 0,25 & 0,58 \\
\hline \multicolumn{3}{|c|}{ DESVIO PADRÃO\% } & & 73,54 & 0,22 & 0,25 & 0,48 & 147,08 & 0,11 & 0,13 & 0,24 \\
\hline \multicolumn{3}{|c|}{ MÉDIA PONDERADA } & & 119,00 & 0,74 & 0,61 & 1,35 & 238,00 & 0,37 & 0,30 & 0,68 \\
\hline
\end{tabular}

porte público e a localização do empreendimento afetam diretamente a geração de viagens por pessoas.

Na região de Icaraí, o desvio padrão encontrado é bem superior a $110 \%$ com menos de 20 pontos e, neste caso, o ITE (2004) recomenda que se amplie o número de condomínios investigados.

Apesar da pequena amostra e da recomendação do ITE (2004), pode-se concluir, de forma exploratória, que a localização do empreendimento tem influência na geração de viagens, tanto por automóveis como para viagens de pessoas. A região mais adensada, onde há facilidade de acesso ao comércio e aos serviços, as taxas de viagens geradas por automóveis são menores que a as taxas na região com pouca densidade habitacional e mais longe do centro. Inversamente, na região mais próxima do comércio existe a preferência pela caminhada e pelo uso do transporte público, enquanto, na região mais distante, o uso do automóvel é mais freqüente, em relação à quantidade de residências.

\subsection{Modelos e equações de regressão}

Para elaborar os modelos de regressão, as observações geradas a partir das contagens em cada região entram como dados. Com o uso do software de análise estatística Minitab ${ }^{\circledR}$, são elaborados modelos de regressão linear relacionando o número de viagens (entradas + saídas) com as variáveis unidades residenciais (UR) e número de vagas (NV).

Para a região de Icaraí, os modelos de regressão linear são considerados bons em relação à variável número de vagas. Os valores de $\mathrm{R}^{2}$ estão acima de $76 \%$, o que é considerado aceitável, de acordo com a metodologia do ITE; porém, em relação à variável unidades residenciais, o $\mathrm{R}^{2}$ é de $62 \%$, abaixo do desejável, mas o desvio padrão é inferior a $110 \%$, permitindo, segundo o ITE(2004), o uso da taxa média ponderada para o cálculo da estimativa de viagens. Apesar de poder estimar o número futuro de viagens, é recomendável que nessa região seja ampliado o número da amostra.

A região de Pendotiba apresenta um desempenho mais equilibrado em relação às variáveis, tanto quanto ao número de vagas (NV), como de unidades residenciais (UR). As equações de regressão obtidas com os dados coletados mostram que $97 \%$ das observações

Tabela 7. Equações de regressão linear

\begin{tabular}{|c|c|c|c|c|c|c|}
\hline \multicolumn{7}{|c|}{ Região de Icaraí } \\
\hline \multicolumn{4}{|c|}{ variável UR } & \multicolumn{3}{|c|}{ variável NV } \\
\hline viagens & Equação & $\mathrm{R} 2$ & $\begin{array}{c}\text { Desvio } \\
\text { padrão(\%) }\end{array}$ & Equação & $\mathrm{R} 2$ & $\begin{array}{c}\text { Desvio } \\
\text { padrão(\%) }\end{array}$ \\
\hline dia todo(6:30 às 20:00) & $3,09+1,354$ (UR) & 0,62 & $69,64 \%$ & $20,43+11,60(\mathrm{NV})$ & 0,81 & $30,35 \%$ \\
\hline manhã & $-8,35+0,5756$ (UR) & 0,60 & $13,67 \%$ & $-3,29-0,511(\mathrm{NV})$ & 0,85 & $6,45 \%$ \\
\hline tarde & $11,44+0,7782$ (UR) & 0,61 & $17,02 \%$ & $23,73+0,6489(\mathrm{NV})$ & 0,76 & $10,93 \%$ \\
\hline \multicolumn{7}{|c|}{ Região de Pendotiba } \\
\hline \multicolumn{4}{|c|}{ variável UR } & \multicolumn{3}{|c|}{ variável NV } \\
\hline viagens & Equação & $\mathrm{R}^{2}$ & $\begin{array}{c}\text { Desvio } \\
\text { padrão(\%) }\end{array}$ & Equação & $\mathrm{R}^{2}$ & $\begin{array}{c}\text { Desvio } \\
\text { padrão(\%) }\end{array}$ \\
\hline dia todo(6:30 às 20:00) & $84,35+3,876$ (UR) & 0,98 & $120,75 \%$ & $84,35+1,938$ (UR) & 0,98 & $60,38 \%$ \\
\hline manhã & $46,41+1,591$ (UR) & 0,96 & $36,35 \%$ & $46,41+0,796(\mathrm{UR})$ & 0,96 & $18,18 \%$ \\
\hline tarde & $37,94+2,285(\mathrm{UR})$ & 0,98 & $23,33 \%$ & $37,94+1,142(\mathrm{UR})$ & 0,98 & $11,66 \%$ \\
\hline
\end{tabular}


feitas em campo podem ser explicadas pelas equações dos modelos, considerado bom, dentro da metodologia do ITE (2004). Entretanto, pode-se observar que o desvio padrão para viagens diárias na variável UR apresenta valor acima do recomendado. Seguindo o procedimento sugerido pelo ITE, pode-se utilizar a equação de regressão para a estimativa das viagens diárias.

As características de uso do solo, densidade e disponibilidade de serviços e transporte público são determinantes na geração de viagens de um modo geral. Voltando-se à Tabela 3, em que são resumidas as taxas obtidas nos dois estudos, pode-se observar que, de acordo com o manual do ITE, a taxa por Unidades Residenciais apresentada pelo ITE em empreendimentos unifamiliares (estudo 210) é pouco maior que o dobro (numa proporção de 2,3 para 1) em relação com a taxa apresentada para empreendimento com mais de 10 pavimentos (estudo 222). Já a pesquisa em Niterói apresenta que a proporção entre as taxas geradas por Unidades Residenciais entre Icaraí e Pendotiba é de 3,7 para 1, superando o efeito da tipologia indicada pelo ITE, o que pode ser atribuído à localização desses condomínios.

\section{CONCLUSÕES E RECOMENDAÇÕES}

As cidades carecem de mais estudos para a determinação de suas taxas de geração de viagens. Modelos e taxas desenvolvidas e em outros países não refletem a realidade de cidades brasileiras. Os estudos de estimativa de viagens e seus impactos são ferramentas fundamentais para o planejamento de transporte, do sistema viário e uso do solo em todas as cidades.

O objetivo deste trabalho foi definir taxas e modelos de geração de viagem para a realidade de duas regiões na Cidade de Niterói. Para isso, foi desenvolvida uma metodologia simples, que pode ser utilizada em outros locais e não se limita apenas à realidade do estudo. O resultado foi alcançado e esta metodologia de pesquisa pode ser aplicada em outras cidades.

Os modelos apresentaram resultados consistentes, apesar da pequena amostra e da sua natureza exploratória abordarem apenas duas regiões distintas na cidade de Niterói. Não devem ser aplicados automaticamente a outros locais ou outras cidades, onde podem existir diferentes padrões de mobilidade. Portanto, é fundamental que novos estudos sejam realizados, considerando amostras maiores e novas tipologias de áreas e condomínios.

A metodologia utilizada neste estudo pode ser expandida para qualquer lugar e fazer parte de outros trabalhos.

A hipótese investigada nesta pesquisa foi confirmada. Os fatores de localização e infraestrutura interfe- rem na mobilidade do cidadão. As variações de proximidade dos centros de serviços fornecem características diferentes à geração de viagens, apresentando distintos resultados nos comportamentos das pessoas em relação à mobilidade. A facilidade de acesso ao comércio e aos serviços faz com que a região mais adensada produza menos viagens em automóveis e mais viagens a pé por unidade residencial, enquanto a região mais dispersa e longe do centro apresenta característica oposta: mais viagens por automóvel e menos viagens a pé.

As taxas e os modelos de geração de viagens elaborados neste trabalho podem ser o começo para a determinação de parâmetros compatíveis com as especificidades locais que, como o estudo demonstrou, são divergentes da bibliografia encontrada que traduz os padrões americanos de mobilidade.

É essencial para as cidades, portanto, que o fenômeno de geração de suas viagens seja conhecido e mensurado para diferentes tipos de uso do solo. Só dessa forma os planejadores poderão ter a visão das relações entre os PGVs, os centros, as regiões e a malha viária que as conectam, permitindo-lhes interferir de forma mais saudável e qualificada nessas relações.

\section{AGRADECIMENTOS}

O segundo autor agradece ao CNPq pelo apoio no fornecimento das bolsas e ambos os autores, à Rede Ibero-Americana de Estudo em Pólos Geradores de Viagens (http://redpgv.coppe.ufrj.br).

\section{REFERÊNCIAS BIBLIOGRÁFICAS}

Andrade, E. P. e Portugal, L. S. (2010) Checking the validity of the ITE Trip Generation Models for brazilian shopping centers. ITE Journal. 80 (8), 40 - 44. ISSN 0162-8178. Institute of Transportation Engineers. Disponível em http://www.ite.org/

Bruton, M. J. (1979 Introdução ao Planejamento dos Transportes. Rio de Janeiro: Interciência; São Paulo, Editora Universidade de São Paulo.

CET-SP (1983) Companhia de Engenharia de Tráfego de São Paulo, Pólos Geradores de Tráfego. Boletim Técnico 32. Prefeitura de São Paulo.

Cunha, R. F. de F. (2009) Uma Sistemática de Avaliação e Aprovação de Polos Geradores de Viagens (PGVs). Dissertação de Mestrado. PET/COPPE/UFRJ, Rio de Janeiro, Brasil.

Inocêncio, P. e Grando, L. G. (2007) Elaboração de Modelos de Geração de Viagens Terrestres para Hotéis, Anais do XXI Congresso de Pesquisa e Ensino em Transportes, ANPET, Rio de Janeiro, RJ.

IBGE (2009) Instituto Brasileiro de Geografia e Estatística. Disponível em: <www.ibge.gov.br/cidadesat>. Acesso em: 22 jul. 2009.

IRF (2009) World Road Statistics, Geneva.

ITE (2004) Trip Generation Handbook, An ITE Recommended Practice, Washington, D.C.

ITE (2008) Institute of Transportation Engineers - ITE. Trip Generation Handbook, Washington, D.C.

Oliveira, I. C. E. (2001) Estatuto da Cidade, para compreender. Rio de Janeiro, IBAM/DUMA.

Portugal, L. da S. e Goldner, L. G. (2003) Estudo de Pólos Geradores de Tráfego e seus Impactos nos Sistemas Viário e de Transportes, $1^{\mathrm{a}}$. Edição, São Paulo: Editora Edgard Blücher.

REDPGV (2009) Rede Ibero-americana de Estudos em Pólos Geradores de Viagens. Disponível em: <www.redpgv.coppe.ufrj.br>. Acesso em: 12 jul. 2009. 